\title{
Clear cell sarcoma of the kidney in an adult: a case report and literature review
}

\author{
Mingxin $\mathrm{Cao}^{1} \wedge$, Junlong Zhang ${ }^{1}$, Huisi $\mathrm{Ma}^{2}$, Yueyou Liang ${ }^{1}$ \\ ${ }^{1}$ Department of Urology, the First Affiliated Hospital of Sun Yat-sen University, Guangzhou, China; ${ }^{2}$ Department of Pathology, the First Affiliated \\ Hospital of Sun Yat-sen University, Guangzhou, China \\ Correspondence to: Yueyou Liang. Department of Urology, the First Affiliated Hospital of Sun Yat-sen University, Guangzhou, China. \\ Email: lyuey@mail.sysu.edu.cn.
}

\begin{abstract}
Clear cell sarcoma of the kidney (CCSK) in adults is extremely rare. In fact, only 16 adult CCSK cases have been reported from 1989 to 2020 in the English language literature. The pathologic diagnosis of the disease is difficult, and the optimal treatment is still unknown. Currently, no literature review has been done on adult CCSK patients. Herein, we report the case of a 24-year-old man who presented with right flank pain for one month. The patient underwent a series of diagnostic tests, and imaging examinations revealed a large mass in his right kidney. The patient underwent retroperitoneal laparoscopic nephrectomy and regional lymphadenectomy. Pathological examination of the tumor revealed nests and cords of fairly uniform oval cells with clear cytoplasm. Immunohistochemistry showed that the tumor cells were positive for vimentin, CyclinD1, and Bcl-2 and that the sample was uniformly negative for Wilms' tumor 1 (WT1), CD34, desmin, and cytokeratin staining. Based on these histopathological and immunohistochemical results, the patient was diagnosed with CCSK. The patient subsequently refused chemotherapy and radiotherapy. During the 2-year follow-up, no recurrence or metastasis was observed. We reviewed the English language literature on adult CCSK published in the PubMed database. A pooled analysis was performed, and the results suggested that an accurate pathological diagnosis of CCSK could be achieved based on microscopy and immunohistochemistry. Nephrectomy and regional lymphadenectomy are the main treatments for adult patients with CCSK. While the value of adjuvant radiotherapy and chemotherapy remains controversial, multimodal oncologic treatment, including surgery and chemotherapy with or without radiation, may be efficacious in preventing local recurrence and distant metastases.
\end{abstract}

Keywords: Clear cell sarcoma; kidney; adults; case report

Submitted Aug 13, 2021. Accepted for publication Dec 16, 2021.

doi: $10.21037 /$ tcr-21-1629

View this article at: https://dx.doi.org/10.21037/tcr-21-1629

\section{Introduction}

Clear cell sarcoma of the kidney (CCSK) is a rare tumor that accounts for approximately $5 \%$ of all primary renal tumors in children (1). Though its occurrence is extremely uncommon in adults, it has been the subject of isolated case reports. Because of its rarity, there is little information on its clinical and histological features. The pathologic diagnosis of the disease is difficult, and the optimal treatment remains unknown. To the best of our knowledge, there have been no reports of a literature review on adult CCSK. Therefore, in order to increase awareness and contribute to the knowledge base about adult CCSK, we present the case of a 24-yearold man with CCSK. We reviewed the English language literature of this neoplasm in the adult age group and performed a pooled analysis of case data from the literature. This is the first study to focus on the diagnosis and treatment of adult CCSK. We present our case in accordance with the

\footnotetext{
^ ORCID: 0000-0001-6231-0766.
} 

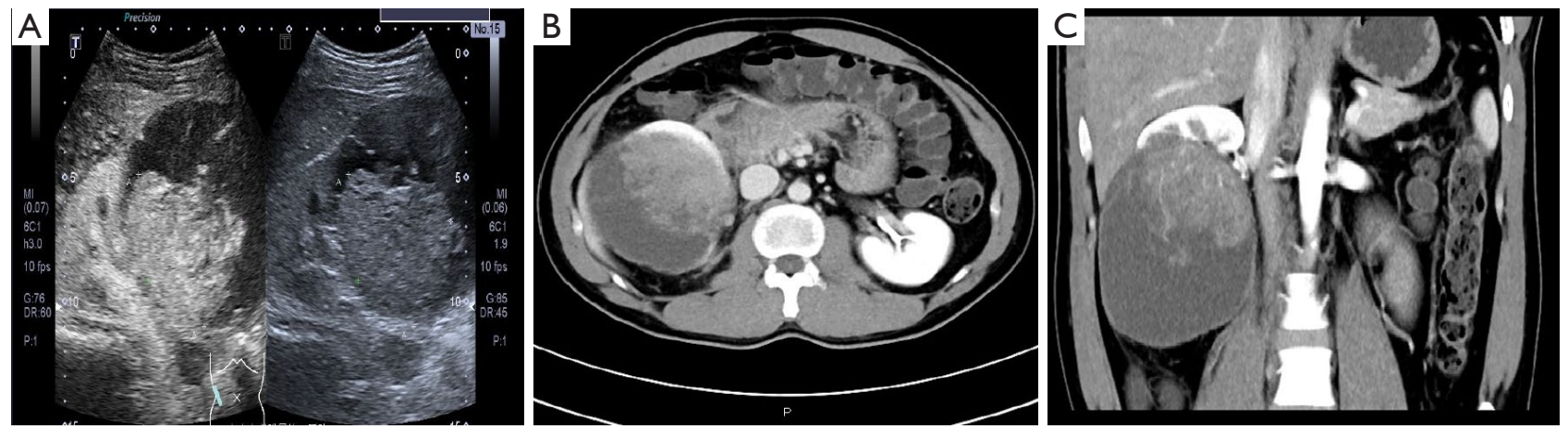

Figure 1 The patient's imaging findings. (A) Color duplex sonography reveals a mixed echo, heterogeneous enhanced mass in the early stage. (B,C) CT shows a large, heterogeneous mass in the middle and lower poles of the right kidney, infiltrating the renal calices. CT, computed tomography.

CARE reporting checklist (available at https://tcr.amegroups. com/article/view/10.21037/tcr-21-1629/rc).

\section{Case presentation}

A 24-year-old man who presented with right flank pain for one month was admitted to our hospital. His family had no related medical history. Physical examination revealed no abnormal findings. The patient's blood chemistry showed elevated levels of serum lactate dehydrogenase (LDH) (278 U/L, normal reference range, 114-240 U/L) and increased plasma D-dimer levels $(3.34 \mathrm{mg} / \mathrm{L}$, normal reference range, $<0.55 \mathrm{mg} / \mathrm{L})$. On color duplex sonography, a round, single, well-circumscribed, mixed echo, heterogeneous mass located in the middle and lower poles of the right kidney was revealed. Dynamic contrastenhanced ultrasonography showed that the mass was uneven and enhanced in the early stage, and was slightly enhanced in the later stage (Figure 1A). The decay time was 60 s. Computed tomography (CT) revealed a large, heterogeneous mass in the middle and lower poles of the right kidney, infiltrating the renal calices (Figure 1B,1C). No signs of metastasis were observed.

The patient underwent retroperitoneal laparoscopic right radical nephrectomy and regional lymphadenectomy. No intraoperative or postoperative complications were observed. The patient recovered uneventfully and was discharged six days postoperatively. Gross examination of the surgical specimen showed a $10.5 \times 10 \times 9 \mathrm{~cm}$, soft, tan-gray mass originating in the parenchyma of the right kidney. The tumor was located adjacent to the renal pelvis and, on cut surface, produced abundant mucinous material with a glistening appearance. It also included a large cyst, areas of hemorrhage, and multiple foci of necrosis. No lymph node metastases were observed. Microscopy revealed nests and cords of fairly uniform oval cells with clear cytoplasm. The tumor cells were separated by arborizing the fibrovascular septa. The nuclei were vesicular and had dispersed chromatin and inconspicuous nucleoli. Necrosis was observed in the tumor tissue. Immunohistochemistry revealed that the tumor cells were positive for vimentin, Bcl-2, and CyclinD1 (Figure 2). Staining for desmin, cytokeratin, CD34, PAX-8, EMA, and Wilms' tumor 1 (WT1) was negative. Based on these findings, the pathological diagnosis of the tumor was confirmed as CCSK, and it was staged as T2bN0M0. The patient subsequently refused chemotherapy and radiotherapy. The patient went into remission 24 months postoperatively.

After searching the PubMed database, we found a total of 16 adult CCSK cases, including 12 male and 4 female patients. These cases were published in the English language literature from 1989 to 2020 . The 16 patients in these studies ranged in age from 19 to 62 years, with a mean and median age of 32 and 25 years. Of the 16 patients, 8 had tumors in the right kidney and 8 had tumors in the left kidney. The diameter of the tumors ranged from 6 to $20 \mathrm{~cm}$, with a mean diameter of $12 \mathrm{~cm}$. The patients' presenting symptoms, their primary and subsequent treatments, their follow-up durations, and their follow-up status are listed in Table 1.

All procedures performed in studies involving human participants were in accordance with the ethical standards of the institutional and/or national research committee(s) and with the Helsinki Declaration (as revised in 2013). 
Table 1 CCSK in adults: review of literature-clinical findings, including presenting symptoms, primary and subsequent treatment and follow-up duration, follow-up status

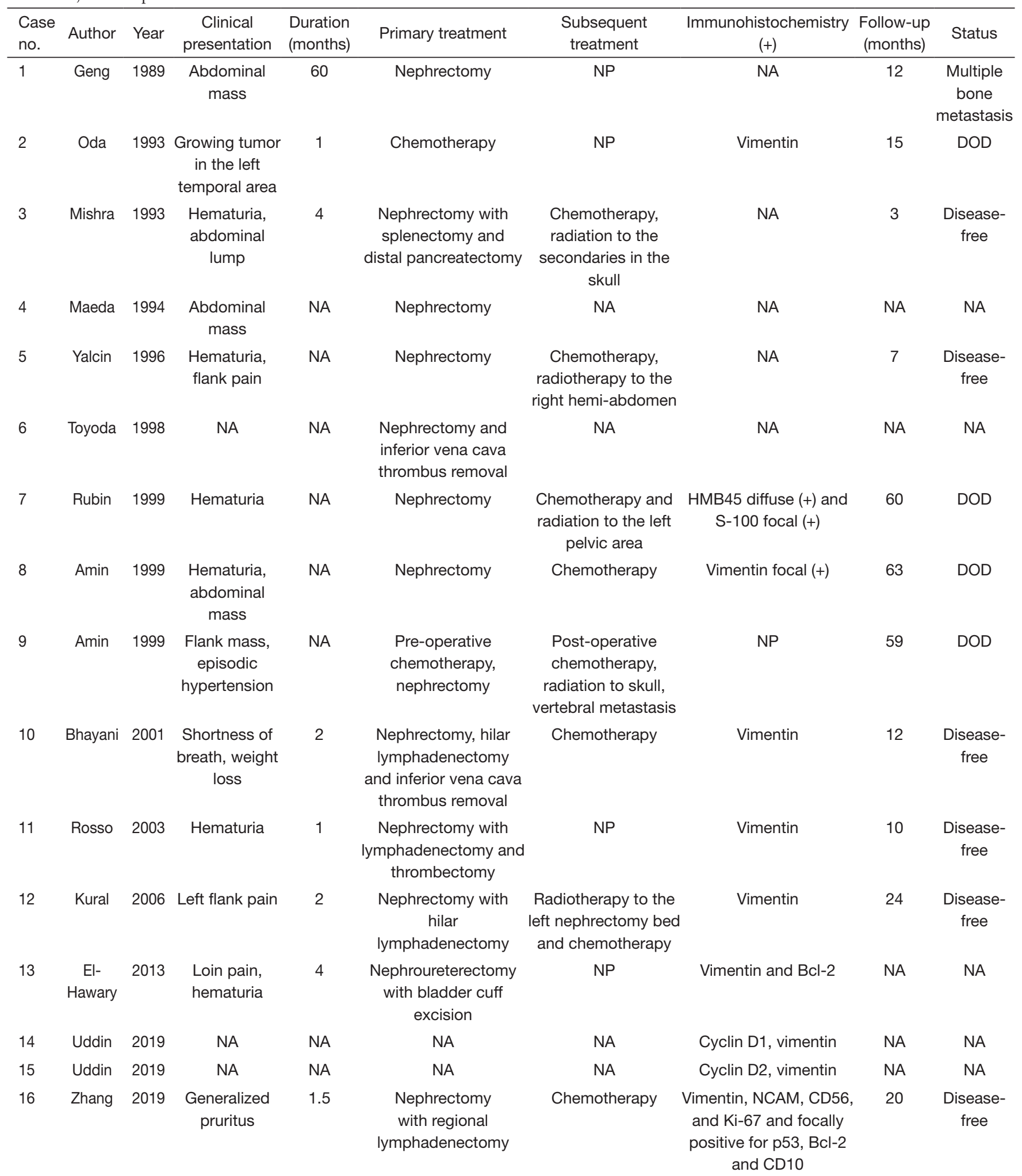

+, positive. CCSK, clear cell sarcoma of the kidney; NP, not performed; NA, not available; DOD, dead of disease. 

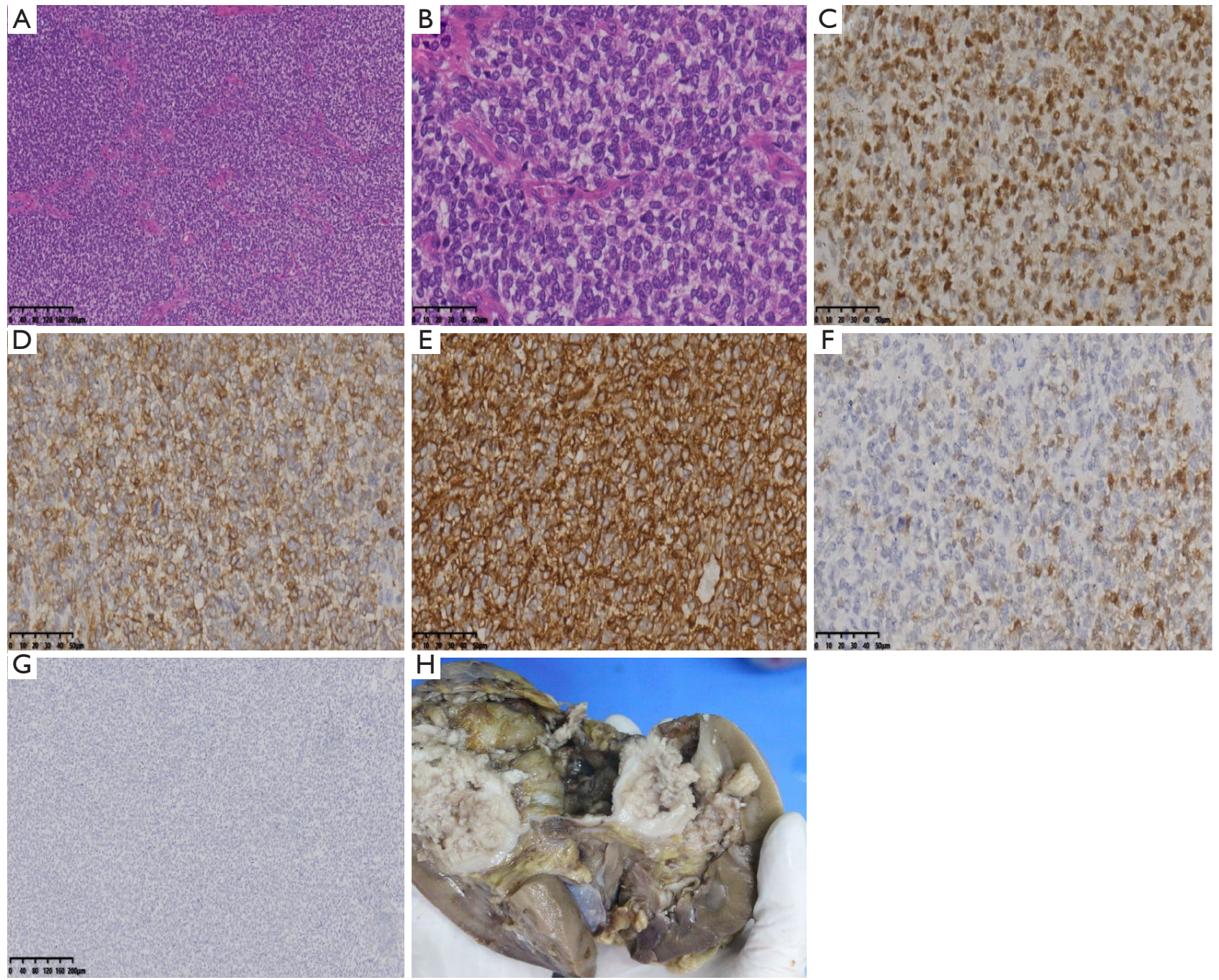

Figure 2 The patient's gross and microscopic pathological images and immunohistochemical results. (A) Loose tumor stroma filled with fibrous vessels with some slightly dense areas (H\&E, $\times 100)$. (B) The classic pattern of CCSK is characterized by round or spindle-shaped cells bundles, fine nuclear chromatin, pale cytoplasm, and unclear cell borders. The cells form nests separated by a fibrovascular stroma (H\&E, ×400). (C) Diffuse positive nuclear staining for Cyclin D1 in the tumor cells (magnification, $\times 400)$. (D) Diffuse cytoplasmic and membranous immunoreactivity for Bcl-2 (magnification, $\times 400$ ). (E) Vimentin coreceptor immunohistochemistry reveals diffuse nuclear staining (magnification, $\times 400$ ). (F) S-100 highlights focal areas in the nucleus (magnification, $\times 400$ ). (G) Desmin staining of the tumor cells in the cytoplasm (magnification, $\times 100$ ). $(\mathrm{H})$ The gross appearance of the mass. CCSK, clear cell sarcoma of the kidney.

Written informed consent was obtained from the patient for publication of this case report and accompanying images. A copy of the written consent is available for review by the editorial office of this journal.

\section{Discussion}

We report a case of CCSK in an adult presenting with flank pain. After a review of the literature in PubMed, we found that there were only 16 adult CCSK cases reported in the English language literature from 1989 to 2020. The subsequent discussion is based on the cumulative data from 17 cases (16 reported cases and 1 current case).

CCSK had been considered to be a more malignant histological variant of WT. In 1970, it was recognized as a separate clinicopathologic entity by Kidd because of its 
potential ability to metastasize to the bone and the brain (2). Marsden et al. (3) named the tumor, "the bone metastasizing renal tumor of childhood", and the term, "clear cell sarcoma" was first used by Beckwith and Palmer.

As in children, CCSK tends to occur predominantly in male adults (4). In fact, in the 17 adult cases found in this study, the ratio between male and female patients was 13:4. The disease duration in these patients ranged from 1-60 months (with a median of two months). Common clinical presentations included hematuria (six cases) and abdominal/flank masses (five cases). Other symptoms included hypertension (one case), metastatic temporal area mass (one case), and weight loss (one case). Zhang et al. (5) reported a case presenting with generalized pruritus. They believed that the pruritus in their patient was caused by paraneoplastic itch. Although in a series of pediatric cases, there is a left-sided preference for CCSK tumors (6), this pattern was not observed in the adult group found in this study. To date, there have been no reports of bilateral kidney involvement in adult patients.

The gross appearance of adult CCSK tumors is often soft and tan-grey in color. Furthermore, the tumor glistens on cut surface due to the tumor's excretion of numerous mucinous materials. As shown in our case, the sacs with necrotic, or hemorrhagic areas are often present. Since the sacs lack distinct capsules with an epithelial lining, they are pseudocysts, filled with mucus and necrotic tissue. In four cases, gross extension into the inferior vena cava occurred, and the tumor extended into the right atrium in two cases (7-10). Microscopically, tumor cells are arranged in nests or cords. The traditional pattern of CCSK is marked by round/elliptical cells with clear cytoplasm, fairly uniform round/elliptical and colorless nuclei with frequent vesicles, finely dispersed chromatin, inconspicuous nucleoli, and unusual mitotic phenomena (11). The tumor surrounds isolated nephrons, with an obvious vascular network and abundant collagenous extracellular matrix materials (4). In general, we found that the pathologic features of adult CCSK are very similar to those of pediatric patients.

Immunohistochemistry should be used to facilitate a differential diagnosis. Of the 17 adult CCSK patients, 11 had immunohistochemistry data. Ten cases stained positive for vimentin, which is consistent with most literature $(12,13)$. In fact, vimentin positivity is a strong pathological characteristic of CCSK. Bcl-2, Cyclin D1, and S-100 staining were positive in three, two, and two cases, respectively. HMB45, cyclin D2, CD56, Ki-67, P53, $\mathrm{CD} 10$, and desmin staining showed variable nonspecific positivity. The specific immunohistochemical characteristics of CCSK in adults remain unclear. Recently, some studies have reported that Cyclin D1 and Bcl-6 coreceptor $(B C O R)$ antibodies help discriminate CCSK from a number of pediatric renal neoplasms. Cyclin D1 has been demonstrated to be sensitive, but not entirely specific for CCSK $(14,15)$. Cyclin D1 staining has also been reported to be very useful for discriminating between CCSK and blastema-rich WT (16). Diffuse strong nuclear staining using a commercially available $B C O R$ antibody has been proven to be highly sensitive and specific for the diagnosis of CCSK in relation to pediatric renal neoplasia (17). However, the value of Cyclin D1 and BCOR antibodies for the diagnosis of CCSK has not been sufficiently verified in adult patients. To date, there are no definite, specific, immunohistochemical indicators that could reliably discriminate CCSK from other renal neoplasms in adult patients. A complete immunohistochemical regimen, including vimentin, cytokeratin, WT1, desmin, Cyclin D1, and BCOR antibodies may be the best choice for determining a final diagnosis. Since CCSK can appear in a variety of histological patterns, the immunohistochemical results should be analyzed by experienced pathologists.

The main differential diagnoses in our case included blastema-rich WT and sarcomatoid variants of renal cell carcinoma. Because CCSK and blastema-rich WT are similar in morphological appearance, distinguishing between these types of neoplasia can be difficult. The most important histological difference between these types of neoplasia is the presence of a moderate amount of cytoplasm in the neoplastic cells of CCSK. In blastemarich WT cells, however, the cytoplasm can barely be visible on histologic examination. The neoplastic cells in our case were uniform and contained abundant amounts of clear cytoplasm. Moreover, the positivity for vimentin and Cyclin D1 staining favors the diagnosis of CCSK, and the negativity for WT1 staining is important for excluding WT. Sarcomatoid variants of RCC are a sign of highgrade transformation in RCC and not a distinct histological entity. It contains both mesenchymal (sarcomatoid) and epithelial (carcinomatous) components (18). The presence of carcinomatous cells can distinguish sarcomatoid variants of RCC from a true sarcoma of the kidney.

The exact molecular pathogenesis and cell of origin of CCSK remain poorly understood. However, some pediatric case reports describe the involvement of chromosomal translocations and other genetic alterations. Ueno-Yokohata et al. (19) found internal tandem duplications in the BCOR 
gene (Bcl-6 corepressor) in 100\% (20/20) of CCSK tumors, and they did not identify these genetic alterations in any $(0 / 193)$ of the other pediatric renal tumors. They believed that $B C O R$ tandem duplication was highly specific to CCSK. Additionally, YWHAE-NUTM2B/E, YWHAE-FAM22, and $I R X 2-T E R T$ gene fusions have been identified in a minority of CCSK cases $(20,21)$. However, most CCSKs display few genomic aberrations, and the underlying mechanism for tumorigenesis still needs to be determined.

The gold standard of care for CCSK remains undetermined, especially in adult patients. In the studies, we found that all 17 adult patients with CCSK underwent total nephrectomy. For patients with inferior vena cava involvement, a surgical thrombectomy was performed. Toyoda et al. and Bhayani et al. $(8,10)$ independently reported two different patients who had the same procedure done. Both patients underwent radical nephrectomy and inferior vena cava surgical thrombectomy through cardiopulmonary bypass and circulatory arrest. In addition to surgery, most of the 17 adult CCSK patients received multimodal oncologic treatment, including chemotherapy with or without radiation. Radiotherapy was administered to five patients. Preoperative chemotherapy was administered to one patient, and postoperative chemotherapy was administered to eight patients. Different combinations of chemotherapy regimens, including doxorubicin, actinomycin D, cyclophosphamide, and vincristine, have been reported (9). The 5-year overall survival rate in pediatric patients improved with the use of modern chemotherapy regimens, particularly addition of anthracycline agents, such as doxorubicin (22). Data concerning the use of modern regimens in adult patients are not yet available. There are also reports of the use of surgery alone without radiotherapy and chemotherapy to treat CCSK. Rosso et al. (9) reported that CCSK is highly resistant to radiation and chemotherapy. In our case, the patient refused post-operative chemotherapy and radiotherapy. There was no recurrence at the 2-year followup appointment. Due to the extreme rarity in adult CCSK cases, it is difficult to design a randomized controlled study to confirm the value of adjuvant chemotherapy and radiotherapy. The optimal treatment for adult CCSK remains an area of active investigation.

In conclusion, the pathologic features of adult CCSK are identical to those in pediatric CCSK cases. A combined immunohistochemical regimen, including vimentin, cytokeratin, WT1, desmin, Cyclin D1, and BCOR antibody staining, is useful for final diagnosis. The primary treatment for adult CCSK includes nephrectomy and regional lymphadenectomy. Furthermore, multimodal oncologic treatment, including surgery and chemotherapy with or without radiation, may be efficacious in preventing local recurrence and distant metastases. However, due to the rarity of this condition, further studies, especially joint studies on experimental and clinical trials for adult CCSK, are still needed to demonstrate the molecular pathogenesis and outcome improvements.

\section{Acknowledgments}

The authors would like to thank the patient and his family, and all members of the study team. We would also like to thank Editage (https://www.editage.cn) for English language editing.

Funding: None.

\section{Footnote}

Reporting Checklist: The authors have completed the CARE reporting checklist. Available at https://tcr.amegroups.com/ article/view/10.21037/tcr-21-1629/rc

Peer Review File: Available at https://tcr.amegroups.com/ article/view/10.21037/tcr-21-1629/prf

Conflicts of Interest: All authors have completed the ICMJE uniform disclosure form (available at https://tcr.amegroups. com/article/view/10.21037/tcr-21-1629/coif). The authors have no conflicts of interest to declare.

Ethical Statement: The authors are accountable for all aspects of the work in ensuring that questions related to the accuracy or integrity of any part of the work are appropriately investigated and resolved. All procedures performed in studies involving human participants were in accordance with the ethical standards of the institutional and/or national research committee(s) and with the Helsinki Declaration (as revised in 2013). Written informed consent was obtained from the patient for publication of this case report and accompanying images. A copy of the written consent is available for review by the editorial office of this journal.

Open Access Statement: This is an Open Access article distributed in accordance with the Creative Commons Attribution-NonCommercial-NoDerivs 4.0 International 
License (CC BY-NC-ND 4.0), which permits the noncommercial replication and distribution of the article with the strict proviso that no changes or edits are made and the original work is properly cited (including links to both the formal publication through the relevant DOI and the license). See: https://creativecommons.org/licenses/by-nc-nd/4.0/.

\section{References}

1. Huang CC, Cutcliffe C, Coffin C, et al. Classification of malignant pediatric renal tumors by gene expression. Pediatr Blood Cancer 2006;46:728-38.

2. Gooskens SL, Furtwängler R, Vujanic GM, et al. Clear cell sarcoma of the kidney: a review. Eur J Cancer 2012;48:2219-26.

3. Marsden HB, Lawler W, Kumar PM. Bone metastasizing renal tumor of childhood: morphological and clinical features, and differences from Wilms' tumor. Cancer 1978;42:1922-8.

4. Argani P, Perlman EJ, Breslow NE, et al. Clear cell sarcoma of the kidney: a review of 351 cases from the National Wilms Tumor Study Group Pathology Center. Am J Surg Pathol 2000;24:4-18.

5. Zhang Y, Li J, Wang Y. Clear cell sarcoma of the kidney in a 62-year-old patient presenting with generalized pruritus. BMC Cancer 2019;19:1034.

6. Sotelo-Avila C, Gonzalez-Crussi F, Sadowinski S, et al. Clear cell sarcoma of the kidney: a clinicopathologic study of 21 patients with long-term follow-up evaluation. Hum Pathol 1985;16:1219-30.

7. Oda H, Shiga J, Machinami R. Clear cell sarcoma of kidney. Two cases in adults. Cancer 1993;71:2286-91.

8. Toyoda Y, Yamashita C, Sugimoto T, et al. Clear cell sarcoma of kidney with tumor extension into the right atrium. J Cardiovasc Surg (Torino) 1998;39:489-91.

9. Rosso D, Ghignone GP, Bernardi D, et al. Clear cell sarcoma of the kidney with invasion of the inferior vena cava. Urol Int 2003;70:251-2.

10. Bhayani SB, Liapis H, Kibel AS. Adult clear cell sarcoma of the kidney with atrial tumor thrombus. J Urol 2001;165:896-7.

11. Balarezo FS, Joshi VV. Clear cell sarcoma of the pediatric kidney: detailed description and analysis of variant histologic patterns of a tumor with many faces. Adv Anat Pathol 2001;8:98-108.

12. Walke VA, Shende NY, Kumbhalkar DT. Renal Clear Cell Sarcoma - Anaplastic Variant: A Rare Entity. J Clin Diagn Res 2017;11:ED10-1.

13. Aldera AP, Pillay K. Clear Cell Sarcoma of the Kidney. Arch Pathol Lab Med 2020;144:119-23.

14. Jet Aw S, Hong Kuick C, Hwee Yong M, et al. Novel Karyotypes and Cyclin D1 Immunoreactivity in Clear Cell Sarcoma of the Kidney. Pediatr Dev Pathol 2015;18:297-304.

15. Mirkovic J, Calicchio M, Fletcher CD, et al. Diffuse and strong cyclin D1 immunoreactivity in clear cell sarcoma of the kidney. Histopathology 2015;67:306-12.

16. Uddin N, Minhas K, Abdul-Ghafar J, et al. Expression of cyclin D1 in clear cell sarcoma of kidney. Is it useful in differentiating it from its histological mimics? Diagn Pathol 2019;14:13.

17. Argani P, Pawel B, Szabo S, et al. Diffuse Strong BCOR Immunoreactivity Is a Sensitive and Specific Marker for Clear Cell Sarcoma of the Kidney (CCSK) in Pediatric Renal Neoplasia. Am J Surg Pathol 2018;42:1128-31.

18. Donskov F. Renal cell carcinoma with non-clear cell histology or sarcomatoid differentiation: recent insight in an unmet clinical need. Ann Transl Med 2021;9:97.

19. Ueno-Yokohata H, Okita H, Nakasato K, et al. Consistent in-frame internal tandem duplications of BCOR characterize clear cell sarcoma of the kidney. Nat Genet 2015;47:861-3.

20. Fehr A, Hansson MC, Kindblom LG, et al. YWHAEFAM22 gene fusion in clear cell sarcoma of the kidney. J Pathol 2012;227:e5-7.

21. Karlsson J, Lilljebjörn H, Holmquist Mengelbier L, et al. Activation of human telomerase reverse transcriptase through gene fusion in clear cell sarcoma of the kidney. Cancer Lett 2015;357:498-501.

22. Furtwängler R, Gooskens SL, van Tinteren H, et al. Clear cell sarcomas of the kidney registered on International Society of Pediatric Oncology (SIOP) 93-01 and SIOP 2001 protocols: a report of the SIOP Renal Tumour Study Group. Eur J Cancer 2013;49:3497-506.
Cite this article as: Cao M, Zhang J, Ma H, Liang Y. Clear cell sarcoma of the kidney in an adult: a case report and literature review. Transl Cancer Res 2022;11(1):288-294. doi: 10.21037/tcr21-1629 